\title{
An intelligent curve warning system for powered two wheel vehicles
}

\author{
Francesco Biral • Mauro Da Lio • Roberto Lot • \\ Roberto Sartori
}

Received: 29 April 2010 / Accepted: 27 July 2010 /Published online: 24 August 2010

(C) The Author(s) 2010. This article is published with open access at Springerlink.com

\begin{abstract}
This article illustrates a novel Curve Warning system for motorcycles which has been developed in the SAFERIDER project (www.saferider-eu.org) of the 7th EU FP, among other Advanced Rider Assistance Systems. The Curve Warning function (CW) described here follows a holistic approach, which combines road geometry, motorcycle dynamics, rider input and riding styles. The warning strategy is based on the correction of longitudinal dynamics derived from a previewed ideal manoeuvre (reference manoeuvre) continuously computed from the actual state of the vehicle. Under normal driving conditions the reference manoeuvre matches the rider's and no correction is needed and no warning is given. But if large differences between actual and ideal accelerations are found the rider is warned to decelerate or brake. As soon as the correct value of deceleration is achieved the warning disappears, improving system acceptability. Warnings are given to the rider via an HMI, which uses a haptic accelerator throttle, a vibrating glove and helmet, and a visual display.
\end{abstract}

Keywords Advanced rider assistance systems · Riding simulator C Curve warning - Optimal preview manoeuvre . Motorcycle

This article explains the Curve Warning concept and mathematics, discusses the implementation aspects and presents pilot tests safely conducted on a top-of-the range riding simulator.

\footnotetext{
F. Biral · M. Da Lio

University of Trento,

Trento, Italy

R. Lot $(\bowtie) \cdot$ R. Sartori

University of Padova,

Padova, Italy

e-mail: roberto.lot@unipd.it
}

\section{Introduction}

Motorcyclists are among the most vulnerable groups of road users and current statistics show that they are involved in fatal crashes 20 times more than car users [1]. The MAIDS study [2] found out that PTW rider error is the primary accident contributing factor in $37 \%$ of all cases. Even though other vehicle drivers are responsible for $61 \%$ of multi-vehicle accidents, PTW riders cause $52 \%$ of the MAIDS fatal accident cases. Moreover, in nearly $30 \%$ of cases it was found that the rider did not have time to complete action to avoid the crash. The above facts suggest that warning the rider of a potential hazard ahead of time may help him/her to take corrective action in time and/or reduce the risk of losing control of the vehicle.

The SAFERIDER project of the 7th EU Framework Program aims at introducing advanced driver assistance systems specifically designed for motorcycles, called "Advanced Rider Assistance Systems" (ARAS). The project schedule includes development of five rider assistance functions, embedded in a unified hardware and software framework, namely Speed Alert, Curve Warning, Frontal Collision Warning, Intersection Support and Lane Change Support. Studies show that curve accidents, which are about $15-17 \%$ of all motorcycle accidents, are mainly due to motorcyclists rather than to other road users. This is a reason to design a curve warning function to help the rider when negotiating the curve. Inexperienced riders will benefit more by this, as they are nearly three times more likely to have this type of accident.

However, the development of a curve support function is not a simple translation of the same ADAS developed for cars $[3,4]$, because Powered Two Wheelers (PTWs) differ in many aspects. Motorcyclists are less willing to accept a system that interferes with motorbike dynamics and personal 
driving style. As PTWs are single-track vehicles, they are intrinsically "unstable" systems; while cornering motorcyclists may traverse the width of the lane, so computation of recommendations that account for driver trajectory in the lane is mandatory for a system that aims at producing useful and acceptable warnings when engaging a curve.

In addition, ARAS are technically challenging because, compared to cars, there is less space for sensors and less power available. Motorbikes also exhibit large roll angles, which makes it more difficult to estimate the vehicle position in the lane.

An initial analysis of past and on-going projects and available devices on the market [5] has shown that not much has been done for the development of such systems for PTWs except at informative level. The forerunners of intelligent systems for motorcycles stem from the Japanese Advanced Safety Vehicle (ASV) initiative, concerned with development of technologies for accident avoidance and crash mitigation. A number of prototype in-vehicle systems have been developed among which Yamaha ASV-2, which conveys warning information (e.g. forward collision, curve speed, speedometer, and navigation), on a visual display on the console and via an earpiece worn by the rider. The Yamaha ASV-2 system also includes a curve speed warning function which is at an initial stage of development.

The ISA (Intelligent Speed Adaptation) [6] is a technology that can assist riders in maintaining legal vehicle speeds on the road. A number of European trials have been carried out to define a functional specification for an ISA appropriate for a motorcycle and assess its functionality and performance as well as user acceptance.

Other relevant project such as SIM [7] and PISA [8] are devoted to the development of active electronic devices (e.g. enhanced anti-lock braking system, traction control and brake-by-wire) for PTWs and/or algorithms to activate passive safety devices such as protective inflatable bags worn by the rider or fitted to the vehicle (for lower limb protection).

The Curve Warning (CW) function described in this paper addresses the above aspects with a novel, unique and holistic approach, which combines road geometry, motorcycle dynamics, rider input and riding styles. The article has an initial section that introduces the concept of the Curve Warning function. A section follows with an overview of the hardware and software architecture. Lastly a section is presented that describes the module integration into a riding simulator and a discussion of the preliminary results.

\section{Curve warning concept}

Accident analysis results show that $15-17 \%$ of all motorcycle accidents occur when negotiating a curve, [2]. The Curve
Warning was thus designed to detect incorrect, insufficient or missing rider action in these situations and to warn him unobtrusively but effectively by suggesting a more appropriate action for correct longitudinal control of the vehicle. A typical scenario is shown in Fig. 1: the PTW vehicle travels on a road with curves and possible danger (hot spots, pedestrian crossings, etc). In this situation, the $\mathrm{CW}$ aims to help the rider to positively and safely negotiate the road ahead.

The CW function calculates, at a frequency of 5-10 cycles per second, a reference "optimal-safe" manoeuvre by predicting speed and roll patterns, processing digital maps, inertial measurement and GPS information. Compared with existing systems, the $\mathrm{CW}$ function presented is not based on a set of heuristic rules, nor does it refer to the legal or to any assumed speed constraint. The $\mathrm{CW}$ function is an example of advanced holistic techniques for optimal nonlinear control [9] which accounts for many aspects of motorcycle dynamics and scenario characteristics.

The reference manoeuvre is calculated, at a frequency of 5-10 cycles per second, with a dynamic optimization approach [10], which includes:

- an appropriate mathematical model of the PTW vehicle dynamics;

- the current dynamic state of the PTW;

- a model of the road geometry and attributes;

- the pattern of rider acceleration and target state at the end of the preview horizon.

The function recognizes inadequate rider manoeuvres from the correction of the motorcycle longitudinal dynamics with respect to the forecast manoeuvre. Based on this a Curve Warning Index that rates the risk level for negotiating the road ahead is calculated which is used to properly warn the rider with a predefined set of HMI devices.

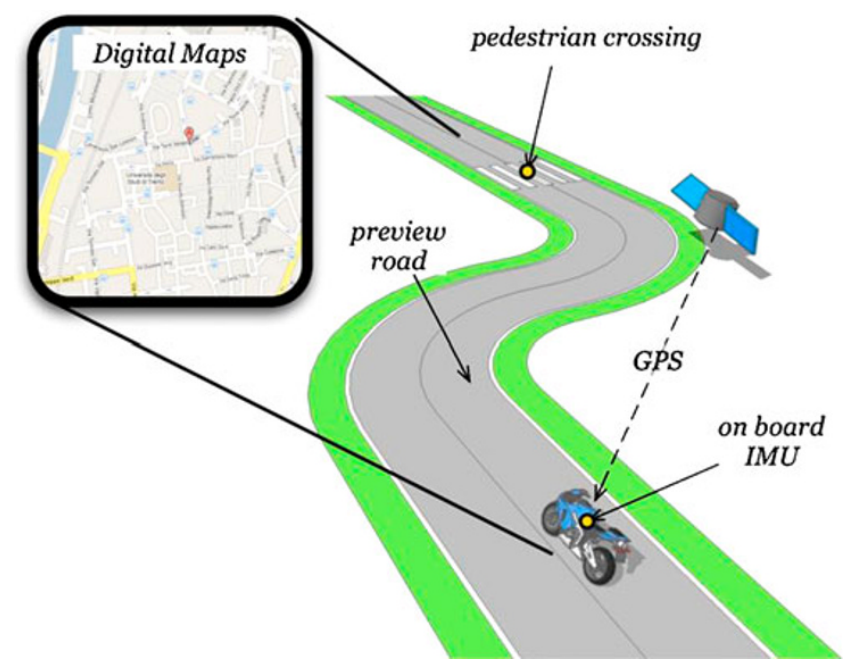

Fig. 1 Typical scenario and main sensors used in the curve warning function 


\subsection{Mathematical formulation}

Calculation of the safe-optimal preview manoeuvre stems from the solution of an optimal control problem that reads as follows: for a given state space model of the vehicle

$$
\dot{\mathbf{x}}=\mathbf{F}(\mathbf{x}, \mathbf{u})
$$

where $\mathbf{x}$ are the state variables and $\mathbf{u}$ are the vehicle controls, find the preview control history u (e.g. brakes, throttle and steering) that minimises a given cost function $J$ (e.g. a combination of riding comfort, distance travelled, etc.) for a given preview time $T$ :

$\min \int_{0}^{T} J(\mathbf{x}, \mathbf{u}) d t$

subject to imposed initial conditions on all state variables

$\mathbf{x}(0)=\mathbf{x}_{0}$

on final condition of selected state variables $\widetilde{\mathbf{x}} \in \mathbf{x}$ :

$\widetilde{\mathbf{x}}(T)=\widetilde{\mathbf{x}}_{T}$

and inequality constraints (i.e. physical limits):

$\mathbf{C}(\mathbf{x}, \mathbf{u}) \leq 0$

The solution of such a problem not only gives the control history $\mathbf{u}(t)$ but also the whole preview motion $\mathbf{x}(t)$ of the vehicle (i.e. trajectory, velocity, roll angle, etc.). The optimal motion predicts how to guide the vehicle smoothly from the current state $\mathbf{x}_{0}$ to a final steady state motion. The preview motion $\mathbf{x}(t)$ also minimises the goal function and keeps, as much as possible, the vehicle state within the safety margin defined by the cost function and inequality constraints.

A specific dynamic optimization algorithm has been developed to solve numerically the resulting non linear system of equations in real time. More details on the adopted approach and numerical algorithm can be found in [10].

\subsubsection{Dynamic model of the PTW vehicle}

Since the calculation of the safe-optimal preview manoeuvre is time demanding but the Curve Warning system must work in real time, an essential, optimized model of the PTW vehicle was developed as described here.

The riding task is quite complex, however in a simple description the longitudinal and lateral dynamics of the vehicle may be considered uncoupled. The rider controls the longitudinal dynamics using throttle and brakes: the most relevant output is the vehicle speed. He controls the lateral dynamics using the handlebar (and secondarily by torso movements): the most relevant output is the vehicle heading.
Based on these considerations, the simplest model that captures the essential motorcycle dynamics is a rigid body controlled in terms of speed and yaw rate and free to roll. In particular, if one imagines this model as a rolling wheel of proper size and inertia, the proposed basic model includes gyroscopic effects and tire shape features that are important in motorcycle dynamics, as is well known (Fig. 2).

The state-space model of the rolling wheel is the following:

$$
\begin{aligned}
\dot{\omega}_{\varphi}= & h \frac{\left(g \sin \varphi-\omega_{\psi} u_{x} \cos \varphi+\omega_{\psi}^{2} h \sin \varphi \cos \varphi\right)}{\rho_{x}^{2}+h^{2}+r h \cos \varphi}+\frac{I_{W}}{m} \frac{\omega_{\psi} \cos \varphi\left(\omega_{\psi} \sin \varphi-u_{x} / R\right)}{\rho_{x}^{2}+h^{2}+r h \cos \varphi} \\
& +r \frac{h\left(\omega_{\varphi}^{2}+\omega_{\psi}^{2}\right) \sin \varphi-\omega_{\psi} u_{x}}{\rho_{x}^{2}+h^{2}+r h \cos \varphi} \\
\dot{\varphi}= & \omega_{\varphi}
\end{aligned}
$$

where the longitudinal speed $u_{x}$ and the yaw rate $\omega_{\psi}$ are the model input and the roll angle $\phi$ and roll rate $\omega_{\varphi}$ are the state variables. Inspection of the first Eq. 1 reveals that the roll rate depends on gravity and centripetal acceleration (1st row), the gyroscopic effect (2nd row), and tire cross section (3rd row).

As discussed above, the basic PTW model can be controlled by the longitudinal speed and the yaw rate; but for smoother motion and better description of riding style, where both reflect human control attitudes [11-13], it is convenient to control the vehicle through jerk (i.e. time derivative of acceleration) instead of speed. Therefore, four additional state variables and equations are introduced as follows:

$$
\begin{aligned}
& \dot{\omega}_{\psi}=a_{\psi} \\
& \dot{a}_{\psi}=j_{\psi} \\
& \dot{u}_{x}=a_{x} \\
& \dot{a}_{x}=j_{x}
\end{aligned}
$$

The road geometry can be synthetically and effectively described using the curvilinear coordinates approach. As shown in Fig. 1, the road centreline may be completely defined by assigning the road curvature $\mathrm{K}$ as a function of the road length $s$, and the position and orientation of the vehicle can be defined using its position $s$ along the route,

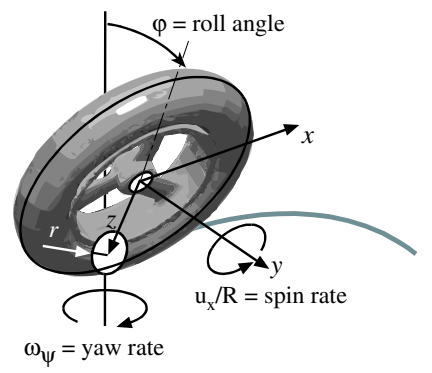

$\begin{array}{ll} & \text { Model parameters } \\ h & \text { center of mass height } \\ m & \text { vehicle and rider mass } \\ \rho_{x} & \text { roll inertia radius } \\ g & \text { gravity acceleration } \\ R & \text { rolling radius } \\ r & \text { tire cross section } \\ I_{W} & \text { spin inertia of wheels } \\ u_{x} & \text { forward speed }\end{array}$

Fig. 2 free rolling wheel model 


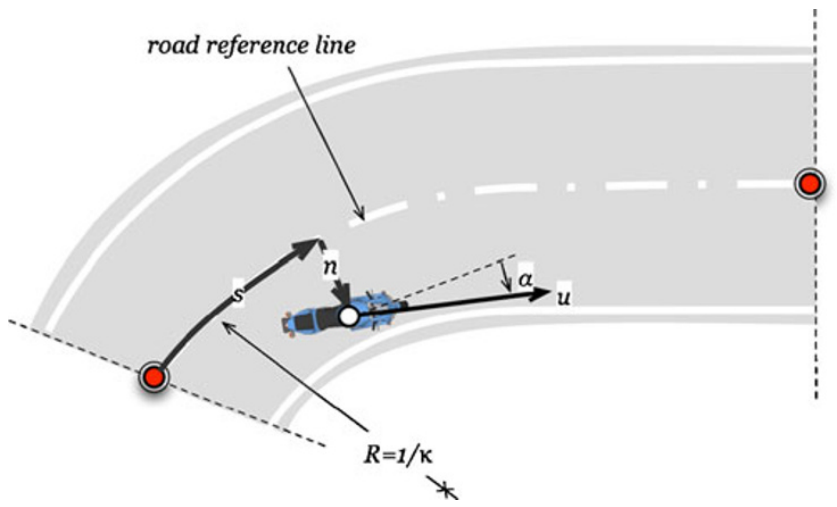

Fig. 3 road description in curvilinear coordinates

the distance $n$ from the road center and orientation $\alpha$ relative to the road direction. This description leads to the following state space model (Fig. 3):

$$
\begin{aligned}
\dot{s} & =\frac{u_{x} \cos \alpha}{n \kappa(s)-1} \\
\dot{n} & =u_{x} \sin \alpha \\
\dot{\alpha} & =\frac{u_{x} \kappa(s) \cos \alpha}{n \kappa(s)-1}+\omega_{\psi}
\end{aligned}
$$

Summarizing, the state space model (1) is composed of Eqs. 6,7 and 8 for a total of nine state variables $\mathbf{x}=$ $\left\{\omega_{\varphi}, \varphi, \omega_{\psi}, a_{\psi}, u, a_{x}, s, n, \alpha\right\}^{T}$ and two inputs $\mathbf{u}=\left\{j_{x}, j_{\psi}\right\}^{T}$

\subsubsection{Cost functions, constraints and boundary conditions}

From the rider's point of view a safe-optimal preview manoeuvre has to satisfy several requirements with a fair margin to cope with possible inaccuracies of the knowledge of their exact values, and must:

a. be consistent with dynamics;

b. satisfy tire adherence limits;

c. stay within the road lane;

d. have steady state motion as the target state at the final preview horizon, guaranteeing motorcycle stability and large manoeuvrability margins if some future, unexpected action is needed (e.g. stop the vehicle, abruptly change direction);

e. if above requirements are satisfied, promote riding comfort and speed.

All these specifications were translated into a mathematical formulation as follows.

Requirement a) was already translated into the state space PTW model (1) along with the imposition of initial conditions (3) $\mathbf{x}(0)=\mathbf{x}_{0}$ according to the real vehicle state.

Requirement $b$ ) for evaluation of tire forces was translated into an equivalent constraint of type [7] on vehicle longitudinal and lateral acceleration that must remain inside an ellipse of diameters $2 a_{x, \max }$ and $2 a_{y \text {,max }}$, i.e.

$C_{1}=\frac{a_{x}^{2}}{a_{x, \max }^{2}}+\frac{u^{2} \omega_{\psi}^{2}}{a_{y_{\text {, } \max }^{2}}^{2}}-1 \leq 0$

Requirement c) translates to a pair of simple inequalities:

$$
\begin{aligned}
& C_{2}=n-b_{R}(s) \leq 0 \\
& C_{3}=-n+b_{L}(s) \leq 0
\end{aligned}
$$

where $b_{R}$ and $b_{L}$ are the lane width respectively on the right and on the left with respect to the road centre that may change with $s$.

Requirement d) translates into final conditions (4) on the following selected state variables:

$\widetilde{\mathbf{x}}(T)=\left\{\begin{array}{c}\omega_{\varphi}(T) \\ a_{\psi}(T) \\ a_{x}(T) \\ n(T) \\ \alpha(T)\end{array}\right\}=\{\mathbf{0}\}$

which corresponds to steady state motion with null roll rate and longitudinal acceleration, with the vehicle at the centre of the lane, whereas the final values of roll angle and yaw
Fig. 4 Experimental examples of rider capability envelope
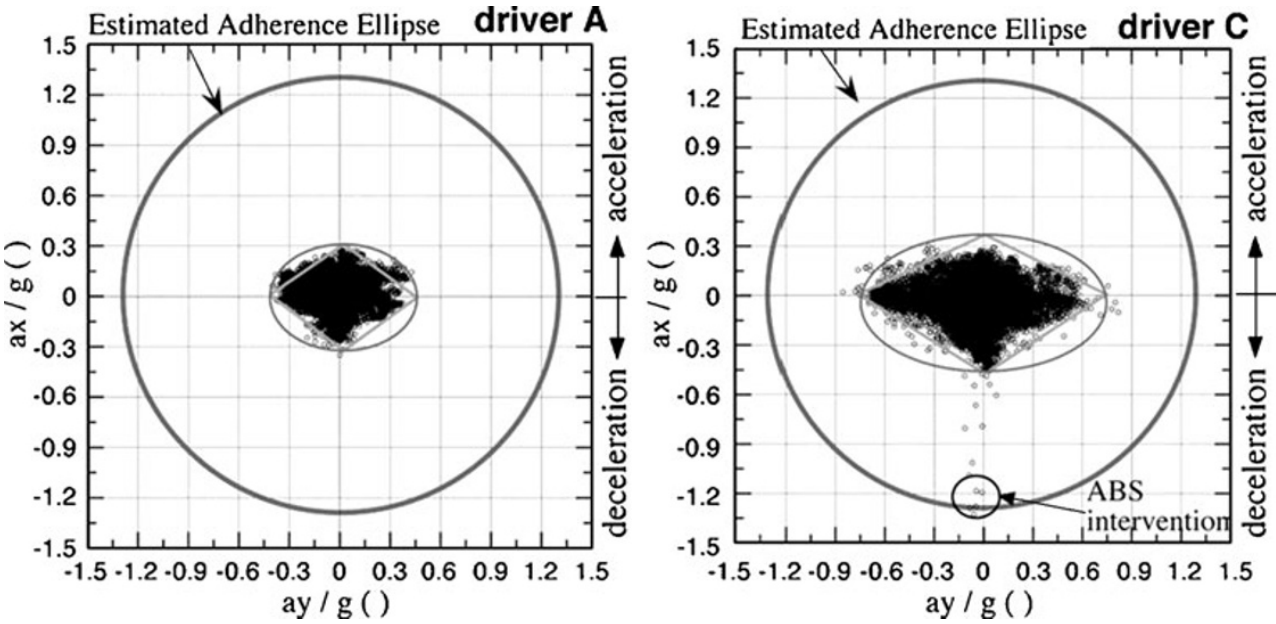
Fig. 5 Comfort cost functions: 3D cost function that implement the capability envelope and 2D function, with double curvature, to implement constraints on jerk.
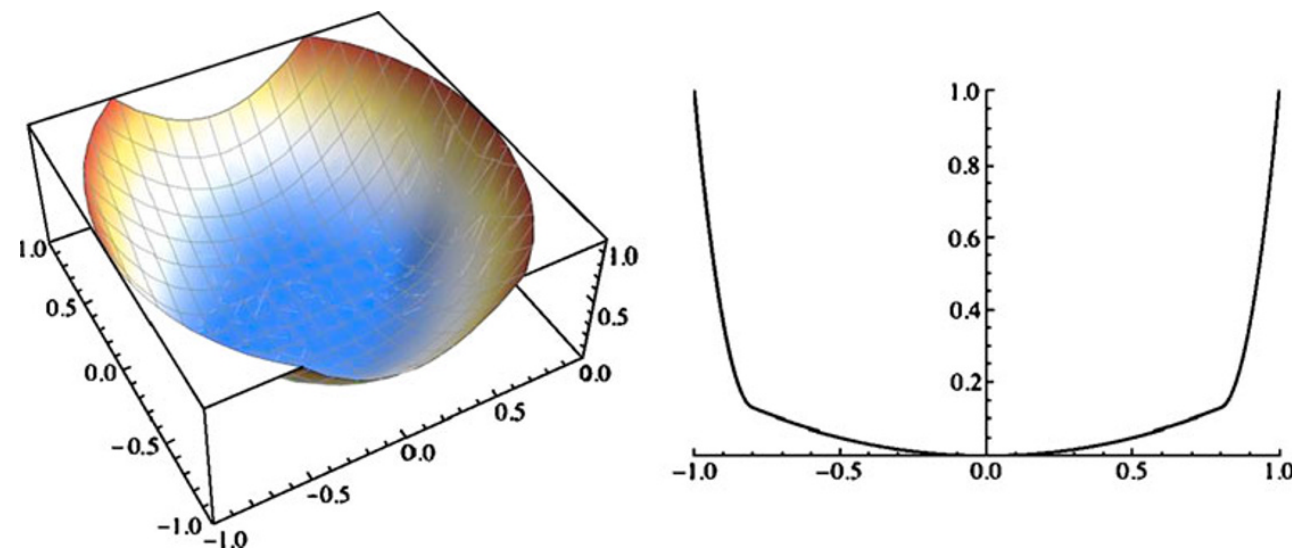

rate are automatically computed consistent with final forward velocity and road curvature.

A first difficulty in including comfort requirements is to define an objective index which quantifies what comfort is for a rider. As a starting point, experimental evidence shows that in normal driving, lateral and longitudinal acceleration values fall within a diamond shape [4, 14], as shown in Fig. 4. The envelope of this pattern was assumed to be the "rider capability envelope" as it encloses the set of states that the rider considers comfortable; moreover this envelope can be easily parameterized to comply with different classes of riders and riding styles. Additionally, many studies show that the rider's command rate of change is limited and occurs at constant jerk [11].

Finally, comfort requirements e) have been introduced into the cost function as follows

$J(\mathbf{x}, \mathbf{u})=W_{a}\left(a_{x}, u \omega_{\psi}\right)+W_{j}\left(j_{x}\right)+W_{j}\left(j_{\psi}\right)-u_{x}$

where $W_{a}$ is the acceleration envelope function and $W_{j}$ is jerk envelopment function, as shown in Fig. 5. The additional term $-u_{x}$ was introduced to promote speed, whenever other requirements are satisfied.

Finally, it is worth noting that comfortable accelerations are fractions of maximum accelerations related to tyre adherence, and therefore are always within safety limits.

\subsubsection{Safe-optimal preview manoeuvre calculation}

Further steps are necessary to perform dynamic optimization of the preview manoeuvre, as explained in detail in reference [10]. Essentially, the time domain mathematical model is converted into a space domain model with the curvilinear abscissa $s$ as the new independent variable and the inequality constraints (5) are converted into penalties to be added to the cost function (12). Both these operations drastically improve the computational efficiency of the optimization algorithm. Finally, Lagrange's approach is used to derive the co-equations of the non-linear optimization problem; the resulting Boundary Value Problem is solved with a specific numerical solver working in real time.
Fig. 6 Preview speed plan and suggested corrective action on longitudinal dynamics. (a)

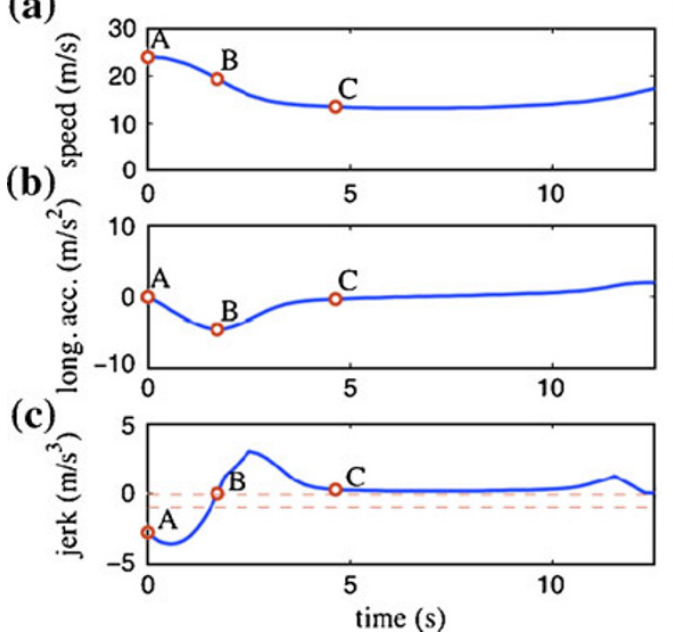

(d)

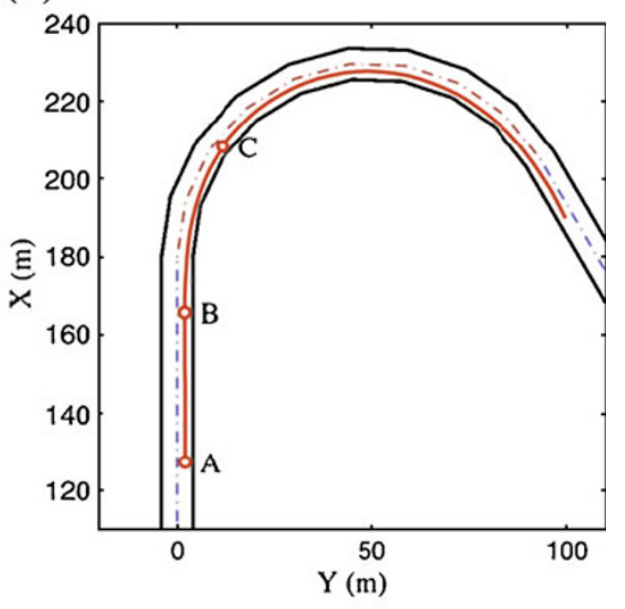


Fig. 7 Sequence of optimal speed plans
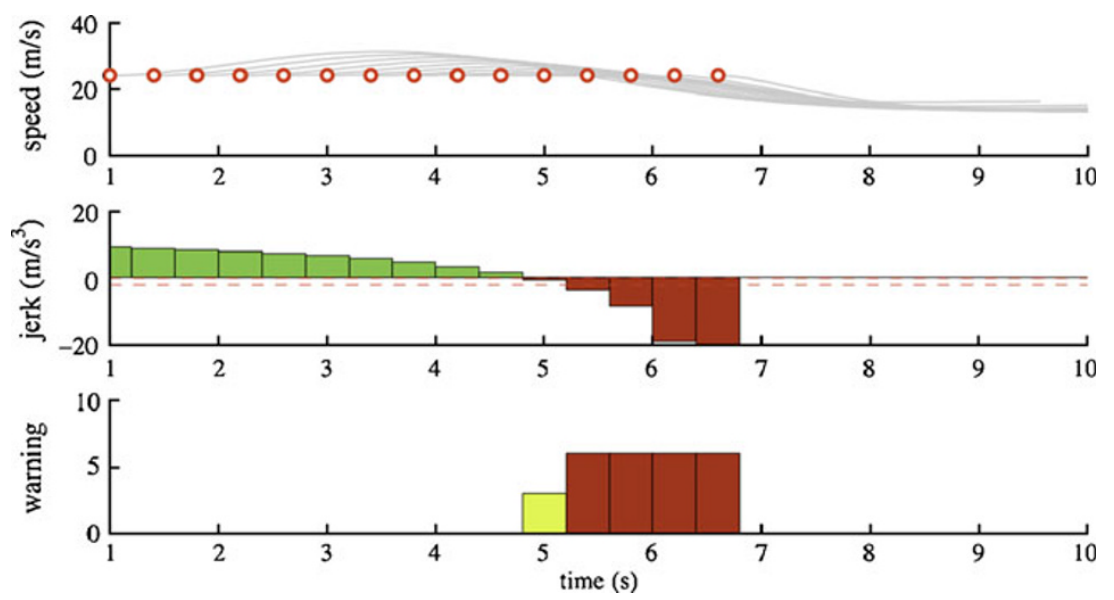

\subsection{Exemplary use of operation}

To better understand the CW concept let us describe how it works under ideal conditions, i.e. when the motorcycle is approaching a curve ahead as illustrated in Fig. 1. Based on real road geometry and the current vehicle state the $\mathrm{CW}$ function computes a preview of the evolution of vehicle dynamics (i.e. velocity, lateral and longitudinal acceleration, roll angle etc.) at the maximum speed compatible with the fixed safety and comfort requirements. Figure 6 shows respectively a) the speed and b) the acceleration profiles calculated from their given initial values. Figure 6a shows that the preview speed initially increases (due to the initial acceleration), then decreases and reaches its minimum in the middle of the curve and finally it increases again at the curve exit.

The calculated preview manoeuvre is just one among the possible paths round the curve ahead and in particular it represents the fastest manoeuvre that complies with the given specifications for safety and comfort. Therefore, if the rider is actually riding faster, or accelerating more than the preview manoeuvre, he/she is potentially in danger and the $\mathrm{CW}$ provides a warning. The potential dangerous behaviour is identified on the basis of preview jerk (i.e. the time derivative of the acceleration, Fig. 6c: as the jerk becomes more negative, the urgency of reducing acceleration (or decelerating even more) increases, therefore two jerk thresholds have been selected for cautionary and imminent warning. As the essence of the preview concept, the first instants of the manoeuvre are strongly influenced by what is next; therefore it is sufficient to examine first values of jerk to suggest what the rider should do now to be in a safer condition later. So, a major benefit of this approach for risk evaluation is that it can provide early warning, e.g. 2-3 s before entering the curve, leaving the rider time to react and correct his behavior.

We note that the warning strategy based on jerk evaluation does not only recognize a possible danger situation, but also evaluates the mismatch between rider

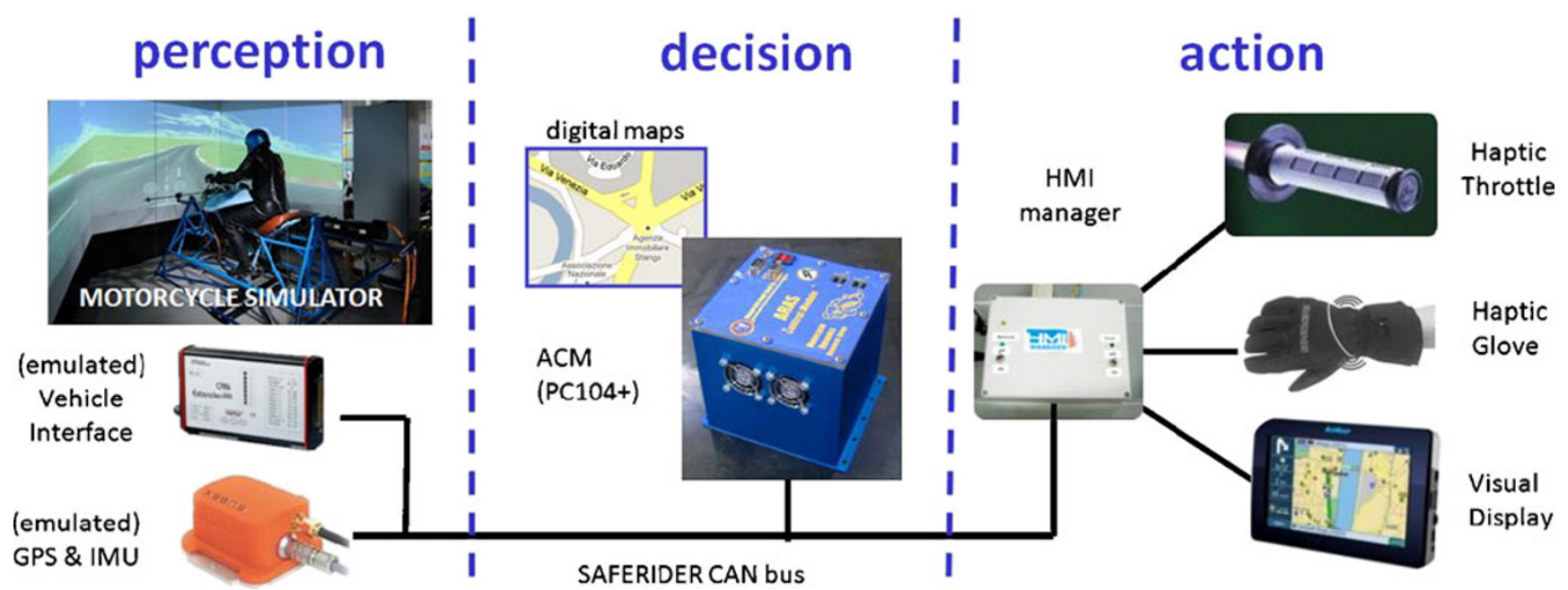

Fig. $8 \mathrm{CW}$ implementation on the UNIPD riding simulator 


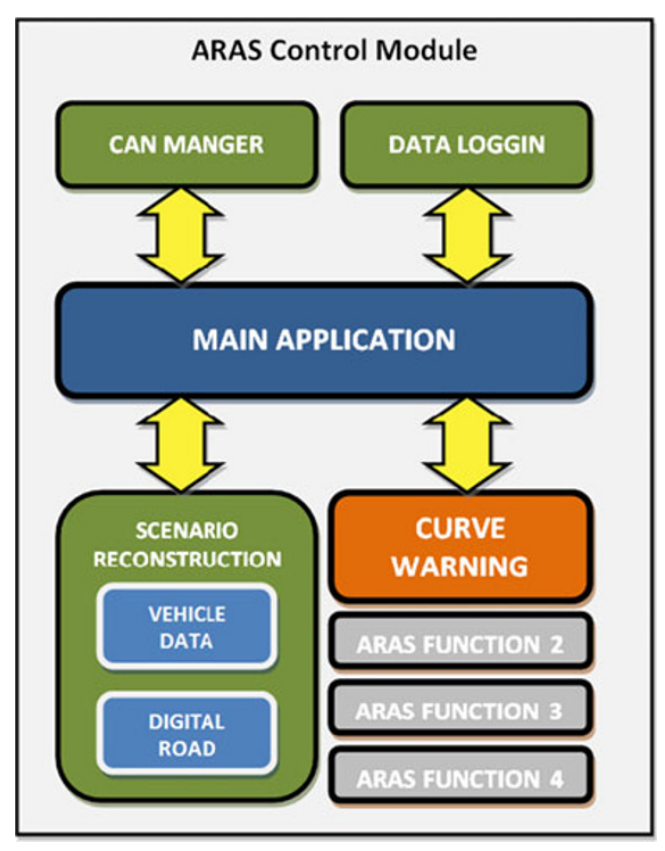

Fig. 9 ACM software architecture

action and system plans, to produce a warning only when there is potential danger and when the rider has not yet seen it. Indeed, in a reference scenario where the rider is at a certain speed and there is a curve ahead, if the vehicle acceleration is null (or even positive), most likely the rider should be warned; on the contrary if the vehicle is decelerating most likely the rider need not be warned because he is aware of the situation and does not need or want a redundant message. The $\mathrm{CW}$ is capable of distinguishing between these situations: in the first case a negative, possibly high, jerk arises in the preview manoeuvre and a warning is delivered, on the contrary in the second situation the preview manoeuvre will be much smoother, with no such negative jerk and hence no warning.

As the rider's behaviour differs from preview manoeuvres, these must be continuously updated to real speed and acceleration, as also to changing road scenario conditions (e.g. road geometry). Consequently as the vehicle approaches the curve, a sequence of manoeuvres is computed as fast as possible (where "fast" is limited by available hardware). Figure 7 shows a rider at constant speed, too high for the next curve. When the curve is at $180 \mathrm{~m}$, no danger is foreseen because there is still time to reduce speed, but as the distance decreases to $60 \mathrm{~m}$, the system warns the rider. Since there are still $2.5 \mathrm{~s}$ before the curve, he/she is still in time to decelerate.

\section{Curve warning implementation}

The first implementation of the CW system was done on a riding simulator. This choice had some important benefits: verification and tuning are carried out in a flexible environment where we can use either real (hardware-inthe-loop) or emulated devices; moreover testing of a safecritical function like $\mathrm{CW}$ in a virtual environment avoids any risk for the user.

The CW system was implemented on the riding simulator of the University of Padua, called Safebike $[16,17]$. Safebike simulator is a top-of-the-range riding simulator built on a mock-up with $5^{\circ}$ of freedom: yaw, pitch and roll angles, lateral position movement and the handlebar steering angle. Riders control the simulator via the throttle, front and rear brake levers, clutch, footrests and the handlebar. The motorbike dynamics, which represents the core of the simulator, is a detailed nonlinear model with $14^{\circ}$ of freedom developed to run in realtime along with a set of possible control systems (digital or mixed analogical/digital) and some hardware sensor emulators.

The CW shares the common ARAS architecture of the SAFERIDER project which is organized in three layersperception, decision and action-reciprocally connected via CAN bus, as shown in Fig. 8.

The perception layer comprises sensors for measurement of vehicle state and, in the simulator integration, includes an emulated GPS device, an emulated Inertial Measurement Unit (IMU), and a Vehicle Interface module (VIF), which emulates the interface to the SAFERIDER CAN bus for vehicle built-in sensors linked to the speedometer, brake pressure and others. On the CAN bus, virtual sensors are indistinguishable from real, due to common timing and protocol.

The action layer consist of the ARAS Control Module (ACM), which manages ARAS software and interacts with

Table 1 Simulator setup for curve warning tests

\begin{tabular}{llllll}
\hline & Acceptance concept & & & Effective concept & \\
\cline { 2 - 3 } \cline { 5 - 6 } & Cautionary & Imminent & & Cautionary & Imminent \\
\hline $\begin{array}{llllll}\text { Tactile } \\
\text { Visual }\end{array}$ & $\begin{array}{l}\text { Haptic throttle low intensity } \\
\text { Constant orange icon }\end{array}$ & $\begin{array}{l}\text { Haptic throttle high intensity } \\
\text { Constant red icon }\end{array}$ & & Vibrating glove low intensity & Vibrating glove high intensity \\
& & & & Constant orange icon & Blinking red icon \\
\hline
\end{tabular}


Fig. 10 Average rider reactions after alarm for each setup: baseline, haptic throttle, haptic glove
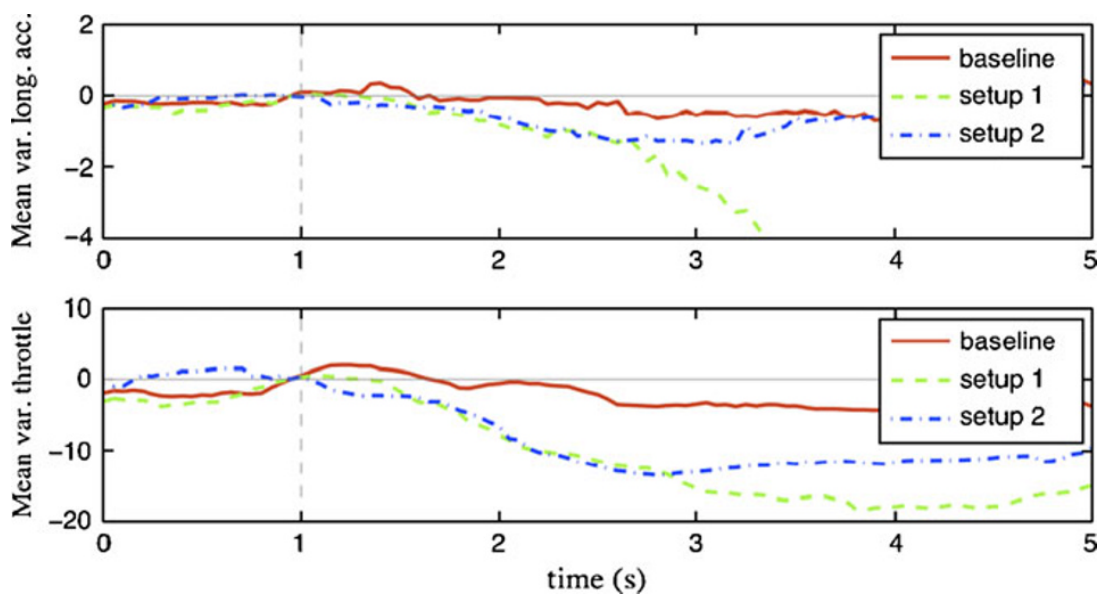

the other SAFERIDER systems, hosted by a PC/104+ with a $1.4 \mathrm{GHz}$ CPU running Linux OS.

Last the action layer includes the HMI manager and a set of HMI elements: the visual display, the haptic throttle and the vibrating glove [18]. The HMI manager processes the warning provided by the ACM and properly activates the various HMI elements. More in detail, the $10^{\prime \prime}$ navigator system display is used to show context-related warning icons; the haptic throttle has an electric motor which increases the resistance torque of the spring as the manoeuvre risk increases, while glove vibration is generated by activating eccentric cylindrical motors via Bluetooth ${ }^{\mathrm{TM}}$.

The CW logic is hosted in the ACM together with other sub-functional modules each of which implements a specific task, as depicted in Fig. 9. The Main Application (MA) is the program in charge of the whole interoperation between modules. The MA implements the high-level ACM logic coordinating the data exchanged between modules and also synchronization of module operation.

The CAN Manager module receives and stores sensor data from the Can bus; then the MA passes this data to the Scenario
Reconstruction (SR) module, which is responsible for the so called "data fusion". This is the computation of heterogeneous sensor data to produce a consistent estimate of the vehicle's state of motion and position with respect the road, based on a digital road model provided by the Digital Road (DR) module. The reconstructed scenario is passed by the MA to the $\mathrm{CW}$ module. Once the warning is generated it is sent back by the MA through the CAN bus at the first opportunity.

While running, the logging module allows the MA program to trace the data exchanged between modules and the state of execution of the whole program. This allows post-process analysis of the entire system behaviour.

\section{Curve warning tests}

A Curve Warning test campaign was conducted with a sample of 20 riders on the SafeBike riding simulator. The test target was to verify the hypothesis that the $\mathrm{CW}$ system enhances riding safety and increases (at least does not decrease) the quality of riding experience. This by evaluation of technical
Fig. 11 Curve warning implementation of the Yamaha Teneré XT660

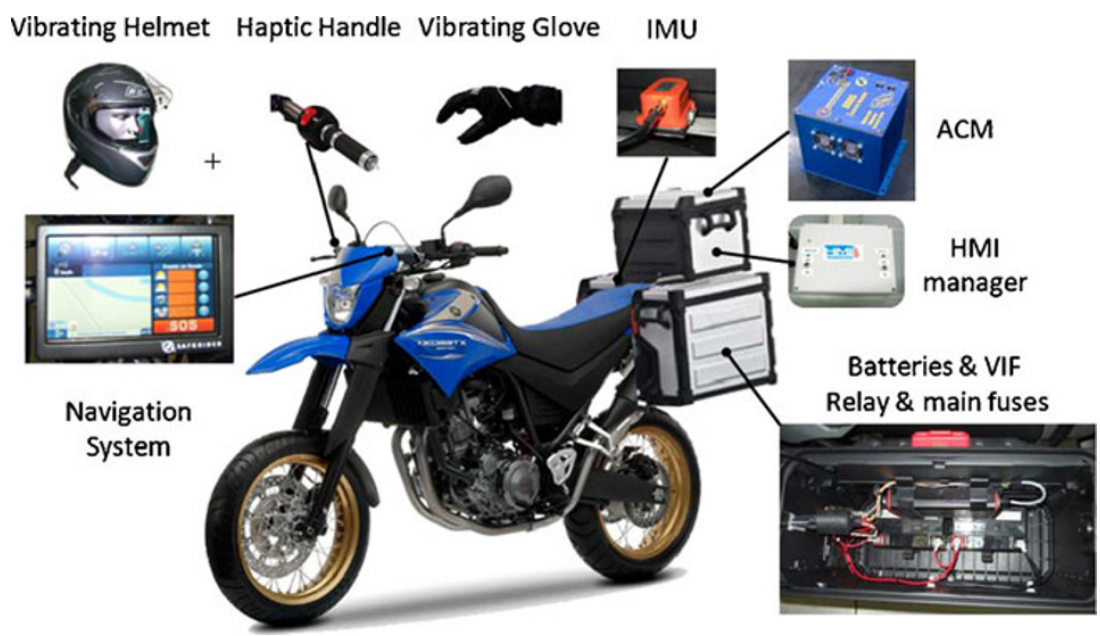


system performance as well as rider usability, workload and acceptance.

Tests were made on two different simulator setups: the first designed to maximize user acceptance and the second to maximize warning effectiveness. As reported in Table 1, each setup includes both a tactile and visual HMI, and there are two warning levels, named cautionary and imminent.

Each test ride was organized in three distinct phases: baseline measurement (CW inactive), acceptance setup and effective setup phase (SAFERIDER systems active). The testing sequence was randomized to counterbalance "ordering-effects", and at the start each test rider had some time to familiarize with the motorcycle simulator. During each phase all relevant system variables and rider commands were logged for objective evaluation, whereas subjective data were collected in interviews and questionnaires at both the start and end of each test ride.

To evaluate objectively the system effectiveness, the rider's throttle commands and the longitudinal acceleration were extracted from logged data for each curve warning event. The longitudinal acceleration was used instead of the brake signals since these were available only as on/off values on CAN bus. Nevertheless, the longitudinal acceleration well represents the overall effect of both throttle and brake pressure variations on the longitudinal dynamics of the vehicle.

For each of the three different setups (baseline, Setup 1 and Setup 2) the throttle and longitudinal acceleration data were extracted $1 \mathrm{~s}$ before the warning alarm and $5 \mathrm{~s}$ after; the average of the data variations after the warning alarm was calculated for all events and all riders with the same setup.

Figure 10 shows the results: each line represents the "average" behaviour that follows a mistake for the three setups. With the baseline (where the $\mathrm{CW}$ is activated to detect critical situations, but no warning is given to the rider) the correction after the alarm is minimal both in term of throttle and acceleration. With the HMI active the riders took stronger corrective action earlier (about $1 \mathrm{~s}$ after the event). However, results do not show any significant difference between the two haptic devices.

We note that the results of Fig. 10 are less significant for values after $3 \mathrm{~s}$ since the related standard deviation is quite high.

Figure 11 shows the integration of the $\mathrm{CW}$ on a motorcycle. ACM, HMI manager, VIF and batteries are in the rear bags; there are several HMI available: Navigation System, Vibrating Helmet, Haptic Handle and Vibrating Glove. In addition, this demonstrator has another three innovative ARAS systems (Speed Alert, Frontal Collision Warning and Lane Change Support).

Altogether the CW system was fitted to two motorcycle simulators and three vehicles within the SAFERIDER project. A more extensive pilot test campaign has been planned, and results will be available within year 2010 .

\section{Conclusions}

The Curve Warning presented is the first attempt to implement a holistic function that warns the rider when he/she is negotiating a curve with incorrect dynamics for the road environment ahead. The warning is generated by calculating an optimal preview manoeuvre that takes into account many aspects: from motorcycle dynamics, to road geometry and characteristics up to riding style and physical limitations.

The CW is one of the Advanced Riding Assistance System under development in the SAFERIDER project, which also includes Speed Alert, Frontal Collision Warning, Intersection Support and Lane Change Support functions. The $\mathrm{CW}$ is based on SAFERIDER architecture and is hosted on an ECU which collects data from available sensors, reconstructs the riding scenario, calculates the preview manoeuvre and provides an appropriate Curve Warning. This warning is subsequently used by the HMI manager to drive the most appropriate HMI element.

The CW function was first tuned and tested on a toplevel riding simulator. The same environment was used by a sample of 20 drivers to evaluate the system with logged objective data and to assess its acceptability via questionnaires. A preliminary objective analysis of the system effectiveness was carried out by calculating the mean variation of longitudinal acceleration and throttle after a warning event. Results show that when the system is active the riders react quickly and with stronger corrections.

The results prove the system can provide a reasonably early warning.

In the framework of SAFERIDER project, the $\mathrm{CW}$ was fitted to two simulators and three vehicles and further driver tests are planned.

Aknowledgment The authors wish to thank the EU for funding this project within the 7th Framework Program and also all other partners involved in the project.

Open Access This article is distributed under the terms of the Creative Commons Attribution Noncommercial License which permits any noncommercial use, distribution, and reproduction in any medium, provided the original author(s) and source are credited.

\section{References}

1. http://ec.europa.eu/transport/publications/statistics/doc/2009 energy transport_figures.pdf

2. MAIDS final report2.0.pdf. Available at: http://www.maids-study.eu/

3. Amditis A, Bertolazzi E, Bimpas M, Biral F, Bosetti P, Da Lio M, Danielsson L, Gallione A, Lind H, Saroldi A, Sjögren (2010) A holistic approach to the integration of safety applications: the INSAFES subproject within the European framework Program 6 
integrated project PReVENT. IEEE Transactions on Intelligent Transportation Systems, ISSN: 1524-9050

4. Bertolazzi E, Biral F, Da Lio M, Saroldi A, Tango F (2010) Supporting drivers in keeping safe speed and safe distance: the SASPENCE subproject within the European framework Program 6 integrating project PReVENT. IEEE Transactions on Intelligent Transportation Systems, ISSN: 1524-9050

5. SAFERIDER benchmarking analysis results: http://www.saferidereu.org/benchmark.html

6. ISA: http://www.its.leeds.ac.uk/projects/isa/

7. SAFETY IN MOTION (SIM) European project: http://www.sim-eu. org/

8. PISa European project: http://www.pisa-project.eu/

9. Bryson AE Jr (1999) Dynamic optimization. Addison-Wesley. ISBN 0-201-59790-X

10. Bertolazzi E, Biral F, Da Lio M (2007) Real-time motion planning for multibody systems. Multibody Syst Dyn 17:119-139, ISSN: 1384-5640, doi:10.1007/s11044-007-9037-7

11. Yamakado M, Abe M (2006) Examination of voluntary driving operational timing by using information obtained with the developed jerk sensor. World Automotive Conference FISITA2006, paper number F2006V137

12. Hiraoka T, Kunimatsu T, Nishihara O, Kumamoto H, Modeling of driver following behavior based on minimum-jerk theory. Proc. of 12 th
World Congress on Intelligent Transport Systems, CD-ROM (2005.11)

13. Biral F, Da Lio M, Bertolazzi E (2005) Combining safety margins and user preferences into a driving criterion for optimal controlbased computation of reference maneuvers for an ADAS of the next generati. In: IEEE Intelligent Vehicle Symposium, June 6-8

14. Biral F, Lot R (2009) An interpretative model of g-g diagrams of racing motorcycle. In: Proceedings of the 3rd ICMEM International Conference on Mechanical Engineering and Mechanics. Beijing, Repubblica Popolare Cinese, Ottobre 21-23, vol 2

15. Biral F, Bortoluzzi D, Cossalter V, Da Lio M (2002) Experimental study of motorcycle transfer functions for evaluating handling. Veh Syst Dyn, ISSN: 0042-3114

16. Cossalter V, Lot R, Sartori R, Massaro M (2008) A motorcycle riding simulator for the improvement of the rider safety. FISITA 2008, paper F2008-11-015, Munich

17. Cossalter V, Doria A, Lot R, Maso M (2006) A motorcycle riding simulator for assessing the riding ability and for testing rider assistance systems. Proceedings DSC 2006. Europe, pp 49-58, Paris

18. Fontana M, Diederichs F, Bencini G, Baldanzini N, Lot R, Sartori R, Spadoni A, Bergamasco M (2010) SAFERIDER: haptic feedback to improve motorcycle safety. Proceedings of the 2010 Haptics Symposium, March 25-26, Waltham 